DOI: $10.4274 /$ gulhane.galenos.2021.26349

Gulhane Med J 2022;64:19-26

\title{
Dermatology consultations in patients with hematological and solid organ malignancies
}

\section{(1) Pelin Eşme, (1) Sema Nur Çoban, (1) Gülşen Akoğlu}

University of Health Sciences Turkey, Gülhane Training and Research Hospital, Clinic of Dermatology and Venereology, Ankara, Turkey

Date submitted:

25.08.2021

Date accepted:

04.12.2021

Online publication date: 15.03.2022

\section{Corresponding Author:}

Pelin Eşme, M.D., University of Health Sciences Turkey, Gülhane Training and Research Hospital, Clinic of Dermatology and Venereology, Ankara, Turkey pelinnesme@gmail.com

ORCID:

orcid.org/0000-0001-6339-2185

Keywords: Dermatology, consultation, hematology, oncology, malignancy

\begin{abstract}
Aims: Patients with hematological or solid organ malignancies are susceptible to various skin disorders. This study described the cutaneous problems related to the underlying diseases or their management in hematology and oncology inpatients.

Methods: In this retrospective study with a cross-sectional analysis, we examined the medical records of inpatients with hematologic or solid organ malignancy who consulted the dermatology department from January 2018 through March 2021. Sociodemographic characteristics, medical history and comorbidities, cancer type, and dermatological findings and diagnoses were noted. Patients who have consulted the outpatient clinic and those with inadequate medical records were excluded.
\end{abstract}

Results: The study included 200 patients (age, mean \pm standard deviation, minimum-maximum: 51.4 \pm 18.2 , 18-89 years; female 51\%). Most of the consultations were from the internal medicine clinic (26.4\%), followed by the hematology and oncology clinics (12.5\%). A quarter of the patients had acute myeloid leukemia, the most frequent disease among hematological malignancies. Breast cancer (7\%) and testis cancer (7\%) were the most common solid organ malignancies. The most common dermatological diagnoses were cutaneous infections (33.5\%) and drug reactions (13.5\%). Skin biopsies were performed in $19.5 \%(n=39)$ of the patients. The evaluation of the patient by a dermatologist for a cutaneous symptom or finding led to modifications of disease management in 67 patients (33.7\%).

Conclusions: Dermatology consultations are frequently required in hematological or solid organ malignancies. A successful evaluation of skin manifestations in these patients may improve the quality of care in this vulnerable population.

\section{Introduction}

Hospitalized patients frequently need multidisciplinary care. Dermatologists may play a pivotal role in managing inpatients, usually more complicated and challenging than many outpatients (1). A study examining 591 dermatology consultations showed that $78 \%$ of the inpatients were misdiagnosed by non-dermatologists (2). Dermatology consultations change the diagnoses made by the primary team in more than $60 \%$ of the patients and affect the treatment plan of most $(1,3-$ $5)$. Despite some discrepancy among the published studies, the most frequent requesting service is internal medicine, while hematology and oncology are among the most common subspecialties $(4,6)$.
However, dermatology is among the top five consultations requested from oncology clinics (7). Due to underlying diseases, chemotherapy, stem cell transplantation, and multi-drug treatments, immunosuppression makes this group prone to cutaneous conditions $(7,8)$. Recently, there have been promising advances in cancer therapeutics with many new treatment agents, including immune checkpoint inhibitors, associated with cutaneous immune-related side effects (7-10). Therefore, there is an increasing need for closer collaboration between hematology, oncology, and dermatology departments $(7,8)$.

Few studies have described the characteristics of skin findings in detail in hematology and oncology patients so far. In this study, we aimed to 1) categorize the frequent dermatological 
diseases in hospitalized patients with malignancy, 2) analyze the role of dermatologists in diagnosing associated dermatological conditions, and 3 ) demonstrate the effect of dermatologists in the treatment strategies of patients.

\section{Methods}

This was a retrospective, cross-sectional study conducted using the medical records of patients diagnosed with hematologic and oncological malignancies who were referred to the dermatology clinic for cutaneous manifestations. The study included inpatient admissions between January 2018 and March 2021. Patients under the age of 18, not hospitalized, or with insufficient medical history were excluded. The study was approved by the University of Health Sciences Turkey, Gülhane Training and Research Hospital Ethics Board (decision no: 2021/123, date: 06.01.2022).

We used the data from the Department of Dermatology and Venereology of the University of Health Sciences Turkey, a tertiary referral care hospital that provides consultant services for inpatients and outpatients. Sociodemographic and medical information, including cancer characteristics (cancer type, current therapeutics) and dermatological findings, were collected from the electronic medical records and patient charts.

\section{Cutaneous conditions evaluated}

Cutaneous conditions were classified into ten groups according to etiology:

1. Cutaneous infections (viral eruption, cellulitis, herpes zoster, fungal infection, scabies),

2. Drug eruptions,

3. Inflammatory disorders (subtypes of dermatitis),

4. Malignant tumors (cutaneous metastasis or primary skin cancer),

5. Steroid-induced acneiform eruption,

6. Deep vein thrombosis and thrombophlebitis,

7. Chemotherapy skin side effects,

8. Xerosis and pruritus,

9. Graft-versus-host disease (GVHD) and,

10. Other skin conditions.

Skin biopsy results following the dermatologist's evaluation were recorded. Recommendations by the dermatologist and whether the consultation changed the patient's immediate treatment plan were also analyzed.

The accuracy of the prediagnoses, if any, specified by the primary care team was also evaluated.

Additionally, we evaluated the effect of Coronavirus disease-2019 (COVID-19) on dermatology consultations requested for hematology and oncology patients.

\section{Statistical Analysis}

Statistical analyses were performed by Statistical Package for the Social Sciences for Windows version 22.0 (IBM, Armonk, NY, USA). Categorical variables were presented by frequency and percentage. Normally distributed continuous variables were expressed as mean \pm standard deviation. Non-normally distributed continuous variables were expressed as median (interquartile ranges, minimum-maximum).

\section{Results}

\section{Demographics}

A total of 2120 inpatient referrals were made during the study period. Of these, 265 (12.5\%) were from the hematology and oncology clinics. Dermatology consultations were ordered most commonly from the internal medicine clinic (26.4\%), followed by the intensive care unit (9.5\%) and surgical clinics (8.3\%). Among the internal medicine clinics, the highest number of referrals were from the hematology and oncology clinics (12.5\%), followed by rheumatology $(6.5 \%)$ and nephrology $(4.7 \%)$.

The number of total consultations requested by years was 42 in 2018 (21\%), 45 in 2019 (22.5\%), 81 in 2020 (40.5\%), and 53 in the only first three months of 2021 (26.5\%). About half of the consultations $(48 \%)(n=96)$ were requested during the COVID-19 pandemic, corresponding to the last 12 months of the 39-month study period. Of the 265 hematology-oncology consultations requested in total, 65 were follow-up consultations of the same patients. Of these patients, $37.5 \%$ had at least one medical comorbidity (Table 1).

\section{Characteristics of underlying malignancies}

Various hematologic $(n=113)$ or solid organ malignancies $(n=87)$ were encountered (Table 1). Distant organ metastases were present by $7.5 \%$. The overall mortality rate was $2 \%(n=4)$. Among the deceased patients, orolabial herpes $(n=2)$, contact dermatitis, and cutaneous metastasis of testis cancer were the dermatological diagnoses following consultations. These patients died within one week of the consultation request.

\section{Role of the dermatology consultation}

In this study, 47 different dermatological diagnoses were made in 10 major disease categories. All consultations were evaluated within $8 \mathrm{~h}$ of the consultation request.

The most common diagnoses were cutaneous infections $(33.5 \%)$ and drug eruptions $(13.5 \%)$, followed by dermatitis $(11.5 \%)$, cutaneous metastasis $(7.5 \%)$ (Figure $1 \mathrm{a}, 1 \mathrm{~b})$, xerosis and pruritus $(5.5 \%)$, steroid-induced acne $(5 \%)$, deep vein thrombosis and thrombophlebitis (\%3), chemotherapy skin side effects (\%3) (Figure 1c, 1d), GVHD (2.5\%), and miscellaneous skin disorders (11.5\%) (Table 2). 


\begin{tabular}{|c|c|}
\hline Age, years, mean \pm standard deviation (minimum-maximum) & $51.4 \pm 18.2(18-89)$ \\
\hline$<30$, mean (standard deviation) & $36(18)$ \\
\hline 50-65, mean (standard deviation) & $54(27)$ \\
\hline$>65$, mean (standard deviation) & $51(25.5)$ \\
\hline Hypertension & $28(14)$ \\
\hline Diabetes mellitus & $18(9)$ \\
\hline Cardiac disease & $11(5.5)$ \\
\hline Benign prostate hypertrophy & $6(3)$ \\
\hline Hematologic malignancy $(n=113)$ & $\mathrm{n}(\%)$ \\
\hline \multirow{8}{*}{$\begin{array}{l}\text { Acute myeloid leukemia } \\
\text { Multiple myeloma } \\
\text { Acute lymphoblastic leukemia } \\
\text { Non-Hodgkin lymphoma } \\
\text { B-cell lymphoma } \\
\text { Mantle cell lymphoma } \\
\text { Burkitt lymphoma } \\
\text { Other (chronic myeloid leukemia, intestinal T-cell lymphoma, large B-cell lymphoma, natural killer } \\
\text { T-cell lymphoma, follicular lymphoma) }\end{array}$} & $50(25)$ \\
\hline & $18(9)$ \\
\hline & $14(7)$ \\
\hline & $14(7)$ \\
\hline & $4(2)$ \\
\hline & $4(2)$ \\
\hline & $4(2)$ \\
\hline & $5(25)$ \\
\hline Malignant melanoma & $4(2)$ \\
\hline Stomach & $4(2)$ \\
\hline $\begin{array}{l}\text { Other [Ewing sarcoma }(n=3) \text {, ovarian cancer }(n=3) \text {, glioblastoma multiforme }(n=3) \text {, osteosarcoma, } \\
\text { peripheral nerve sheath tumor, cholangiocellular cancer, renal cell cancer, osteosarcoma, } \\
\text { liposarcoma, hepatoblastoma, hepatocellular cancer, bladder cancer, rectum cancer, peripheral } \\
\text { nerve sheath tumor, malignant mesenchymal tumor, gastrointestinal stromal tumor, endometrium, } \\
\text { cervix, unknown origin] }\end{array}$ & $25(12.5)$ \\
\hline
\end{tabular}

Drug reaction was the second most common diagnosis. Steven Johnson syndrome (SJS)-toxic epidermal necrolysis (TEN) overlap (Figure 1e) due to the use of phenytoin-valproic acid and fluconazole was observed in two patients with lung and bladder cancers, respectively. Urticarial drug reaction $(n=1)$ and symmetrical drug-related intertriginous and flexural exanthema $(n=1)$ were recorded following administration of ciprofloxacin and piperacillin-tazobactam, respectively. The use of multiple antibiotics ( $\geq 3$ antibiotics simultaneously) was recorded in three patients diagnosed with fixed drug eruptions. In $8(40 \%)$ patients with maculopapular drug eruptions $(n=20)$, simultaneous dual broad-spectrum antibiotic use was recorded; a combination of meropenem-teicoplanin was the most common suspicious dualantibiotic $(n=4)$. Meropenem $(n=7)$, teicoplanin $(n=5)$, piperacillintazobactam $(n=3)$, and trimethoprim-sulfamethoxazole $(n=3)$ were the most common individual suspicious antibiotics leading to a maculopapular (morbilliform) drug eruption. The majority $(73.9 \%)$ of patients with maculopapular drug eruption had a hematological malignancy twice as common in patients with solid organ malignancies ( $13.3 \%$ vs. $5.7 \%$ ).

Hematology and oncology services mentioned a suspicious diagnosis of their own in $34.5 \% \quad(n=69)$ of dermatology consultations but no preliminary diagnosis in $65.5 \%$ of the patients. The dermatology team confirmed the preliminary 


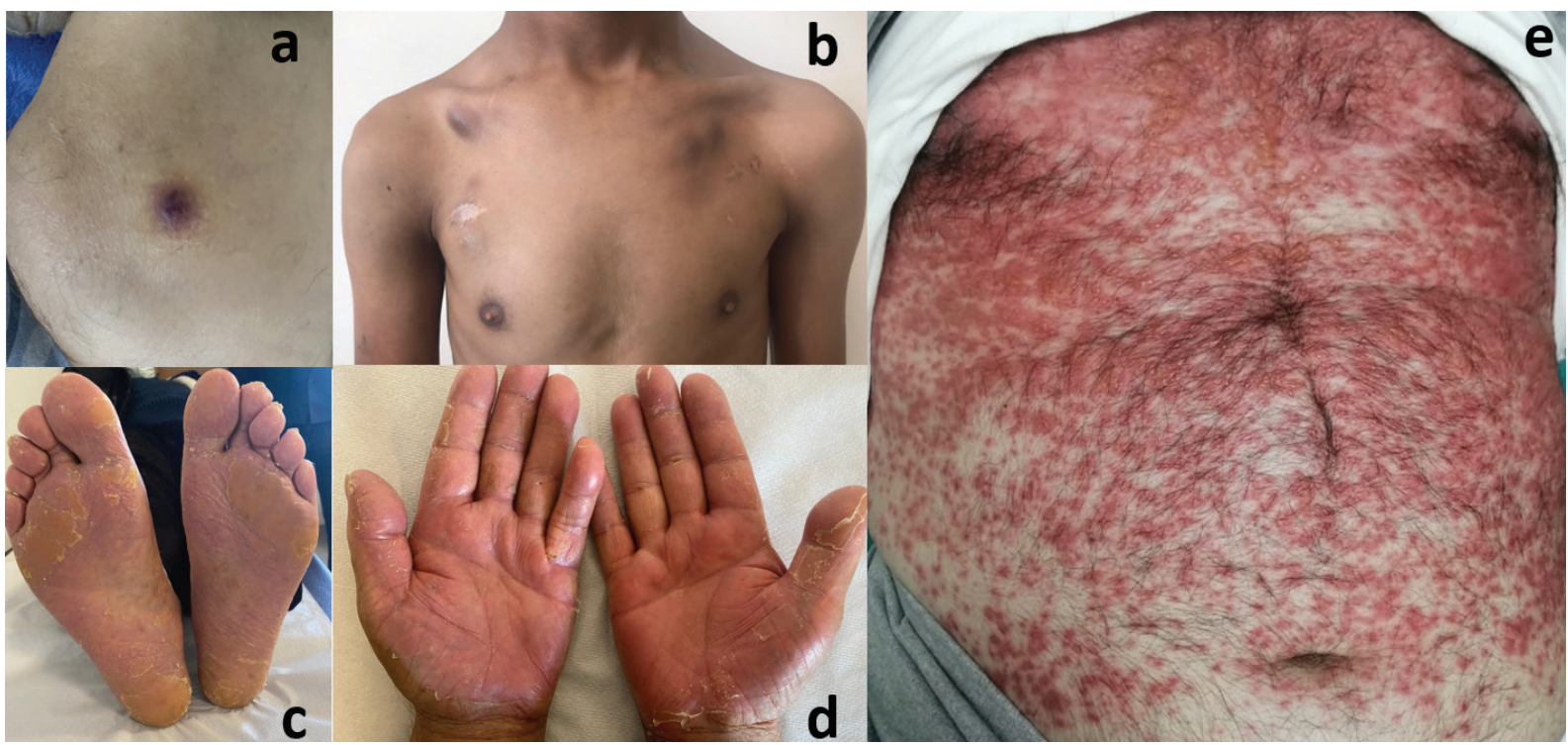

Figure 1. Cutaneous metastasis: a) A 64-year-old male patient with angiosarcoma and bone metastases. Purple-brown nodule, approximately $1^{*} 1 \mathrm{~cm}$ in size, on the medial side of the right knee. Histopathological examination confirmed angiosarcoma skin metastasis. b) A 22-year-old male patient was diagnosed with acute myeloid leukemia. Subcutaneous nodules are darker than normal skin, appearing on the trunk one month ago. The biopsy was compatible with leukemia cutis.

Acral peeling syndrome: c, d). A 76-year-old male patient diagnosed with colon cancer complained of peeling on the hands and feet after the $3^{\text {rd }}$ course of capecitabine treatment. His symptoms were relieved with a potent topical steroid and moisturizer.

Steven Johnson Syndrome-toxic epidermal necrolysis overlap: e) A 68-year-old male patient was diagnosed with prostate cancer (2010) and lung cancer (2019). Fenotion was started due to brain metastases three weeks ago. Nikolsky signs positive areas with the involvement of more than $10 \%$ of the total body. In the early period, intravenous immunoglobulin was planned for the patient. And phenytoin, as well as suspicious other drugs, were stopped immediately

diagnosis by the hematologist or medical oncologist in $70.1 \%$ of 69 patients. The highest diagnostic accuracy was observed in GVHD and cutaneous side effects of cancer therapeutics $(100 \%)$. However, stasis dermatitis was consulted as cellulitis in $30 \%$ of the cases, and $44.4 \%$ of the cutaneous metastases were mistaken for zona zoster or folliculitis. Steroid-induced acne, which can be observed in malignancy patients receiving high-dose steroids according to the treatment schedule, was the least accurately recognized dermatological diagnosis by primary physicians (Table 3 ).

\section{Evaluation of the histopathological results}

Histopathological examination was used in 19.5\% ( $n=39)$ of the patients to confirm the dermatological diagnosis. Dermatology consultations led to changes in the medical management of 63 patients (31.5\%) through the modification of antibiotic treatment $(9 \%)$, current chemotherapy sessions $(17.5 \%)$, or chemotherapeutic options and treatment plans $(5 \%)$. Treatments recommended for the medical management of patients are listed in Table 4.

\section{Discussion}

In this study, the most common cutaneous disorder among dermatology inpatient consultations in hematology and oncology clinics was cutaneous infections followed by drug eruptions. Since oncology and hematology inpatients are immunosuppressed and predisposed to neutropenia due to cancer therapeutics, they are more susceptible to mucocutaneous infections and drug reactions due to multiple drug use and prophylaxis schemes (8-13). Although GVHD $(2.5 \%)$ and cutaneous side effects of chemotherapeutics $(3 \%)$ have been commonly reported in previous studies $(8,10-13)$, they were rarely observed in our study. The current study included only the patients consulted in the dermatology department; therefore, all inpatients in hematology and oncology clinics were not examined. Since our hospital is a tertiary referral center for bone marrow transplantation and cancer treatment, hematologists and oncologists might be more familiar with skin conditions specifically related to their specialties, such as GVHD and the side effects of chemotherapeutics. Therefore, they might have managed some other patients without the need for a dermatology consultation. The correct preliminary diagnoses for these two dermatological diseases also support our suggestion.

The most common diagnoses in our study were cutaneous infections. More than half of them did not relate to any significant risk of mortality and morbidity, such as localized fungal or bacterial infections. The most frequently observed subgroups were zona zoster, cellulitis, and tinea unguium. Interestingly, 


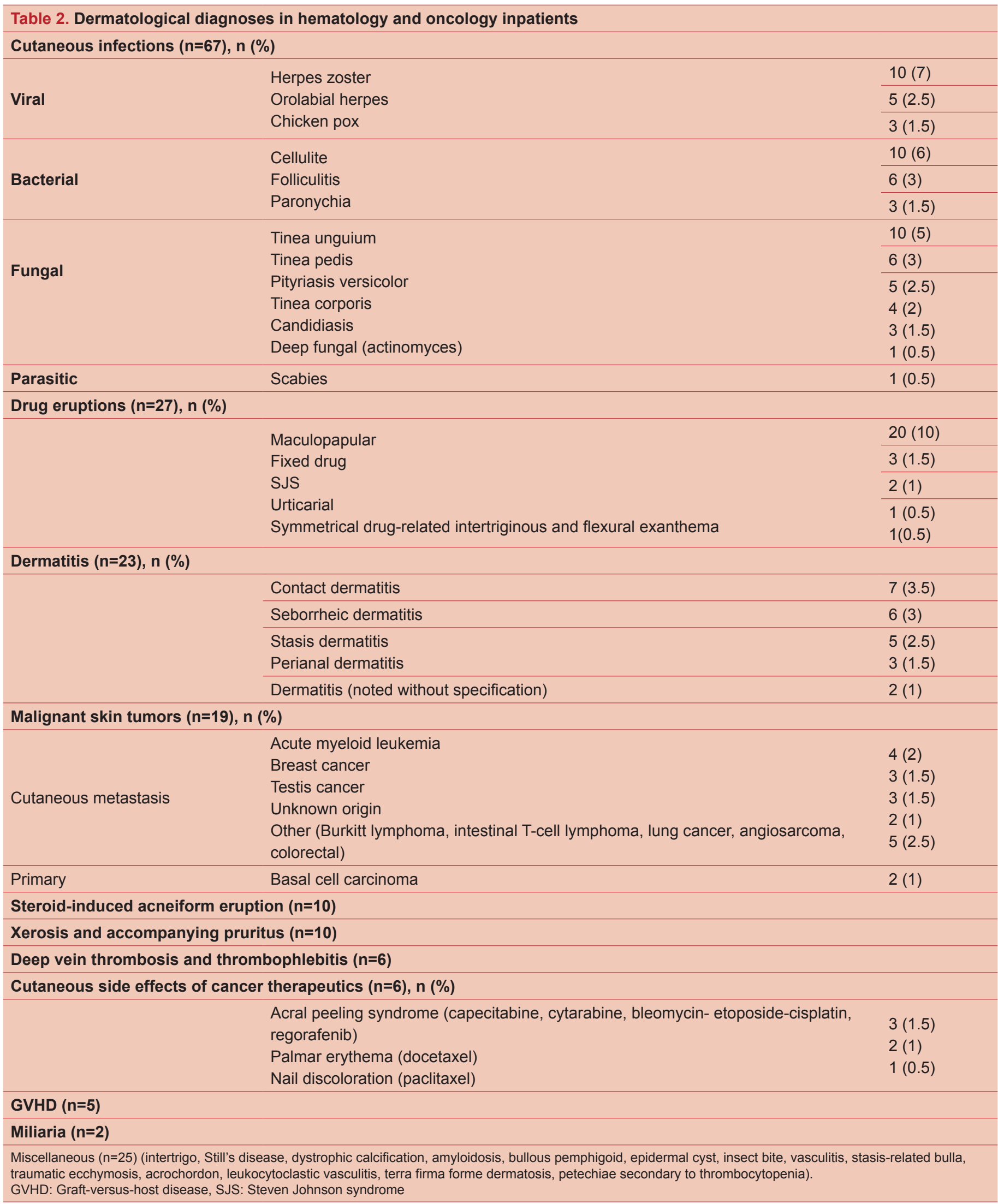




\begin{tabular}{|c|c|c|}
\hline Cutaneous condition & $\begin{array}{l}\text { Consulted with provisional } \\
\text { diagnose/n }\end{array}$ & Diagnostic accuracy $(\%)^{*}$ \\
\hline Graft-versus-host disease & $4 / 4$ & $4 / 4(100)$ \\
\hline Cutaneous side effects of cancer therapeutics & $5 / 6$ & $5 / 5(100)$ \\
\hline Zona zoster & $8 / 10$ & $7 / 8(87.5)$ \\
\hline Tinea pedis and unguium & $5 / 16$ & $4 / 5(80)$ \\
\hline Cellulite & $13 / 15$ & $9 / 13(69.2)$ \\
\hline Drug eruption & $14 / 27$ & $9 / 14(64.2)$ \\
\hline Orolabial herpes & $5 / 5$ & $3 / 5(60)$ \\
\hline Cutaneous metastasis & $9 / 19$ & $5 / 9(55.5)$ \\
\hline Steroid-induced acneiform eruption & $5 / 10$ & $1 / 5(20)$ \\
\hline
\end{tabular}

\begin{tabular}{|c|c|c|c|}
\hline \multicolumn{2}{|c|}{ Topical ( $n=145), n(\%)$} & \multicolumn{2}{|c|}{ Systemic (n=78), n (\%) } \\
\hline Corticosteroids & $55(27.5)$ & Antihistamine drugs & $26(13)$ \\
\hline Emollients & $30(15)$ & Antibiotics & $19(9.5)$ \\
\hline Antibiotics & $25(12.5)$ & Antiviral & $17(8.5)$ \\
\hline Antifungals & $21(10.5)$ & Corticosteroids & $14(7)$ \\
\hline Acne treatments & $10(5)$ & Antifungal & $6(3)$ \\
\hline Wet dressings & $6(3)$ & & \\
\hline Antivirals & $3(1.5)$ & & \\
\hline
\end{tabular}

these infections are some of the essential topics of dermatology education in medical school, which could be better recognized by the primary team and primarily described in the consultation note. Nevertheless, the diagnostic accuracy of cellulitis was relatively low by the primary physicians. Stasis dermatitis was present approximately in one of every three patients consulted dermatology with the diagnoses of cellulitis. One study at two separate centers showed that $20-35 \%$ of patients admitted for cellulitis by the emergency department were mistakenly diagnosed as cellulitis by physicians other than dermatologists and infectious diseases specialists (14). Although stasis dermatitis is a clinical mimicked of cellulitis, bilateral, chronic, and non-tender erythema with usually long-standing pitting edema mainly indicates stasis dermatitis $(1,15)$. A history of trauma in the affected area or accompanying tinea pedis or unguium with tenderness, warmth, swelling, and indistinct borders in a unilateral erythematous plaque, points to cellulitis. Additionally, rapid onset, presence of systemic symptoms such as fever, immunosuppression, and satisfactory response to antibiotics are findings in favor of cellulitis. However, in indistinguishable cases, Doppler ultrasonography could be considered $(15,16)$. These observations suggest that postgraduate dermatology training programs help physicians better assess and manage patients with underlying malignancies and build more proper collaboration with dermatologists.
Drug reactions are another common dermatological condition with a higher risk of morbidity and mortality in patients with underlying malignancies. The frequency of adverse drug reactions in hematology consultations varies between 13 and $38 \%(8,17,18)$. In our study, drug reactions were recorded in $13.5 \%$ of 200 patients, and most of them were morbilliform drug eruptions (10\%) in line with previous literature $9-17.3 \%$ $(8,10,18)$. As in our study, most morbilliform drug reactions were uncomplicated conditions. Morbilliform or maculopapular drug eruptions usually present 1 to 2 weeks after initial exposure of the causative drug, may also show up sooner on rechallenge. Although most cases regress within 1-2 weeks, dermatology consultation is critical in confirming the diagnosis and excluding imitators such as viral exanthema seen in the immunosuppressive patient group $(19,20)$. A dermatologist can help determine the suspected drug(s). More importantly, dermatologists may help to recognize the early signs of severe cutaneous drug reactions (SJS and TEN) such as mucosal involvement, skin tenderness, blistering, dusky red and coalescent macular exanthema, atypical target lesions, and Nikolsky sign (ready removal of the epidermis with slight unrelated pressure) $(20,21)$. Therefore, dermatology consultations should be considered in the early period for patients with diffuse maculopapular eruptions.

The current study demonstrates the possible dermatological diagnoses encountered by physicians working with patients with 
hematology and oncological malignancies. Although 47 different dermatological diagnoses were made in 10 main disease categories, most patients $(72.5 \%)$ were managed with topical therapy, and only $39 \%$ required systemic medications. These data suggested that most skin conditions could be governed without aggressive treatments.

This study presents the 39-month experience of an advanced tertiary center. The last 12 months of the study period corresponds to the COVID-19 pandemic, which has rapidly affected the world and caused severe morbidity and mortality. While our center was mainly in charge of managing patients with COVID-19 infection in this period, hematology and oncology services were at the forefront of the departments that tried maintaining inpatient service properly. In this period, dermatology consultations are essential due to various skin manifestations of COVID-19 infections including urticarial, purpuric, erythema multiforme-like, chickenpox-like rash, acro-ischemia, chilblainlike eruptions, and pityriasis rosea like eruptions (22-25). Intense anxiety among patients and physicians and cutaneous side effects of several systemic medications are likely to increase dermatology consultations during the pandemic period. All these factors are potential causes of the increasing number of dermatology consultations in hematology and oncology inpatients during the pandemic period.

The retrospective design and lack of long-term follow-up are the main limitations of this study. The major strength is documenting a three-year experience of a large population from an advanced tertiary center.

\section{Conclusion}

In conclusion, the current study indicates that dermatology inpatient consultations of hematological or solid organ malignancies play an essential role in determining skin manifestations associated with underlying malignancy or treatment of the disease. A close collaboration between hematologists, oncologists, and dermatologists helps manage patients with underlying malignancies appropriately.

\section{Ethics}

Ethics Committee Approval: The study was approved by the University of Health Sciences Turkey, Gülhane Training and Research Hospital Ethics Board (decision no: 2021/123, date: 06.01.2022).

Informed Consent: Retrospective study.

Peer-review: Externally peer-reviewed.

\section{Authorship Contributions}

Concept: P.E., S.N.Ç., G.A., Design: P.E., S.N.Ç., G.A., Data Collection or Processing: P.E., S.N.Ç., G.A., Analysis or Interpretation: P.E., S.N.Ç., G.A., Literature Search: P.E., S.N.Ç., G.A., Writing: P.E., S.N.Ç., G.A.
Conflict of Interest: No conflict of interest was declared by the authors.

Financial Disclosure: The authors declared that this study received no financial support.

\section{References}

1. Biesbroeck LK, Shinohara MM. Inpatient Consultative Dermatology. Med Clin North Am. 2015;99:1349-1364.

2. Falanga V, Schachner LA, Rae V, et al. Dermatologic consultations in the hospital setting. Arch Dermatol. 1994;130:1022-1025.

3. Storan ER, McEvoy MT, Wetter DA, et al. Experience of a year of adult hospital dermatology consultations. Int J Dermatol. 2015;54:1150-1156.

4. Galimberti F, Guren L, Fernandez AP, Sood A. Dermatology consultations significantly contribute quality to care of hospitalized patients: a prospective study of dermatology inpatient consults at a tertiary care center. Int $\mathrm{J}$ Dermatol. 2016;55:547-551.

5. Milani-Nejad N, Zhang M, Kaffenberger BH. Association of Dermatology Consultations With Patient Care Outcomes in Hospitalized Patients With Inflammatory Skin Diseases. JAMA Dermatol. 2017;153:523-528.

6. Williams A, Bhatia A, Kanish B, Chaudhary PR, Samuel CJ. Pattern of Inpatient Dermatology Consultations in a Tertiary Care Centre from Northern India. J Clin Diagn Res. 2016;10:710.

7. Hoffman M, Smith RJ, Micheletti RG, Rosenbach $M$. Dermatologic support for oncology: Quantifying the consultative services received by hospitalized oncology patients. J Am Acad Dermatol. 2021;85:1367-1368.

8. Uksal U, Ozturk P, Colgecen E, et al. Dermatological Findings in Turkish Paediatric Haematology-Oncology Patients. Eurasian J Med. 2016;48:107-111.

9. Hines A, Hazim A, Wetter DA, et al. Retrospective analysis of 578 inpatient dermatology consultations in hematology and hematopoietic stem cell transplant patients. Int J Dermatol. 2021;60:1385-1391.

10. Chen ST, Molina GE, Lo JA, et al. Dermatology consultation reduces interruption of oncologic management among hospitalized patients with immune-related adverse events: A retrospective cohort study. J Am Acad Dermatol. 2020;82:994996.

11. Alasmari AA, Hakeem AH, Bin Saleh FS, et al. Pattern of Dermatological Disease Encountered in a Hematology Ward: A Retrospective Analysis of Dermatology Consultation in a Hematology Ward in a Tertiary Care Center in Saudi Arabia. Dermatol Res Pract. 2019;2019:9891270.

12. Tracey EH, Forrestel A, Rosenbach M, Micheletti RG. Inpatient dermatology consultation in patients with hematologic malignancies. J Am Acad Dermatol. 2016;75:835-836.

13. Chren MM, Lazarus HM, Bickers DR, Landefeld CS. Rashes in immunocompromised cancer patients. The diagnostic yield of skin biopsy and its effects on therapy. Arch Dermatol. 1993;129:175-181. 
14. Hepburn MJ, Dooley DP, Ellis MW. Alternative diagnoses that often mimic cellulitis. Am Fam Physician. 2003;67:2471.

15. Raff AB, Kroshinsky D. Cellulitis: A Review. JAMA. 2016;316:325-337.

16. Keller EC, Tomecki KJ, Alraies MC. Distinguishing cellulitis from its mimics. Cleve Clin J Med. 2012;79:547-552.

17. Phillips GS, Freites-Martinez A, Hsu M, et al. Inflammatory dermatoses, infections, and drug eruptions are the most common skin conditions in hospitalized cancer patients. J Am Acad Dermatol. 2018;78:1102-1109.

18. Koh $\mathrm{H}$. A retrospective analysis of dermatological problems in a hematology ward. Clin Cosmet Investig Dermatol. 2013;6:145149.

19. Chung WH, Wang CW, Dao RL. Severe cutaneous adverse drug reactions. J Dermatol. 2016;43:758-766.

20. Bircher AJ. Uncomplicated drug-induced disseminated exanthemas. Chem Immunol Allergy. 2012;97:79-97.
21. Scherer K, Bircher AJ. Danger signs in drug hypersensitivity. Med Clin North Am. 2010;94:681-689.

22. Uzuncakmak TK, Bayazit S, Askin O, Engin B, Kutlubay Z. Inpatient dermatology consultations during COVID 19 pandemic in a tertiary referral center. Dermatol Ther. 2020;33:e13883.

23. Wollina U, Karadağ AS, Rowland-Payne C, Chiriac A, Lotti T. Cutaneous signs in COVID-19 patients: A review. Dermatol Ther. 2020;33:e13549.

24. Genovese G, Moltrasio C, Berti E, Marzano AV. Skin Manifestations Associated with COVID-19: Current Knowledge and Future Perspectives. Dermatology. 2021;237:1-12.

25. Daneshgaran G, Dubin DP, Gould DJ. Cutaneous Manifestations of COVID-19: An Evidence-Based Review. Am J Clin Dermatol. 2020;21:627-639. 